\title{
Strategies to distinguish new synthetic cannabinoid FUBIMINA (BIM-2201) intake from its isomer THJ-2201: metabolism of FUBIMINA in human hepatocytes
}

\author{
Xingxing Diao $^{1} \cdot$ Karl B. Scheidweiler $^{1} \cdot$ Ariane Wohlfarth $^{2,3} \cdot$ Mingshe Zhu $^{4}$ • \\ Shaokun Pang ${ }^{5}$ - Marilyn A. Huestis ${ }^{1}$
}

Received: 29 February 2016/Accepted: 9 March 2016/Published online: 28 March 2016

(C) Japanese Association of Forensic Toxicology and Springer Japan (outside the USA) 2016

\begin{abstract}
Since 2013, a new drugs-of-abuse trend attempts to bypass drug legislation by marketing isomers of scheduled synthetic cannabinoids (SCs), e.g., FUBIMINA (BIM2201) and THJ-2201. It is much more challenging to confirm a specific isomer's intake and distinguish it from its structural analog because the isomers and their major metabolites usually have identical molecular weights and display the same product ions. Here, we investigated isomers FUBIMINA and THJ-2201 and propose strategies to distinguish their consumption. THJ-2201 was scheduled in the US, Japan, and Europe; however, FUBIMINA is easily available on the Internet. We previously investigated THJ2201 metabolism in human hepatocytes, but human FUBIMINA metabolism is unknown. We aim to characterize FUBIMINA metabolism in human hepatocytes, recommend optimal metabolites to confirm its consumption, and propose strategies to distinguish between intakes of FUBIMINA and THJ-2201. FUBIMINA $(10 \mu \mathrm{M})$ was incubated in human hepatocytes for $3 \mathrm{~h}$, and metabolites were characterized with high-resolution mass spectrometry (HR-MS). We identified
\end{abstract}

Marilyn A. Huestis

marilyn.huestis@gmail.com

1 Chemistry and Drug Metabolism, IRP, National Institute on Drug Abuse, National Institutes of Health, 251 Bayview Blvd, Suite 200 Room 05A721, Baltimore, MD 21224, USA

2 Department of Forensic Genetics and Forensic Toxicology, National Board of Forensic Medicine, 58758 Linköping, Sweden

3 Department of Drug Research, University of Linköping, 58185 Linköping, Sweden

4 Department of Biotransformation, Bristol-Myers Squibb, Research and Development, Princeton, NJ 08543, USA

5 Sciex, Redwood City, CA 94065, USA
35 metabolites generated by oxidative defluorination, further carboxylation, hydroxylation, dihydrodiol formation, glucuronidation, and their combinations. We recommend 5'OH-BIM-018 (M34), BIM-018 pentanoic acid (M33), and BIM-018 pentanoic acid dihydrodiol (M7) as FUBIMINA specific metabolites. THJ-2201 produced specific metabolite markers 5'-OH-THJ-018 (F26), THJ-018 pentanoic acid (F25), and hydroxylated THJ-2201 (F13). Optimized chromatographic conditions to achieve different retention times and careful selection of specific product ion spectra enabled differentiation of isomeric metabolites, in this case FUBIMINA from THJ-2201. Our HR-MS approach should be applicable for differentiating future isomeric SCs, which is especially important when different isomers have different legal status.

Keywords FUBIMINA - BIM-2201 - THJ-2201 ·

Synthetic cannabinoid · Hepatocyte metabolism - Isomer

\section{Introduction}

Synthetic cannabinoids (SCs) were originally developed as tools to study the endogenous endocannabinoid system [1, 2]. Many $S C$ are $C_{B}$ and $C B_{2}$ receptors agonists, eliciting greater cannabimimetic effects than $\Delta^{9}$-tetrahydrocannabinol [3, 4]. However, SCs marketed as drugs-ofabuse since the 2000s are an important global public health and safety issue [5]. SC drug abuse can produce significant human toxicity including agitation, seizures, hypertension, emesis, myocardial infarction, and even death [6-9]. For these reasons, many SCs were scheduled across the globe, and many more structural analogs emerged. However, little is known about metabolism of these new SCs, which makes it challenging to document their intake in clinical 
screenings, complicated by the fact that the parent SCs rarely are found in urine, the most common analytical matrix.

Compared to structural analogs, it is much more challenging to confirm the intake of isomers of scheduled SCs by mass spectrometry (MS) because the SCs and their major metabolites display identical molecular ions and often identical product ions on a mass spectrometer. FUBIMINA (BIM-2201), BIM-018, AB-CHMINACA, and 1-n-pentyl-3-benzoylindole are isomers of THJ-2201, THJ-018, AB-CHMINACA $2 H$-indazole analog, and 1-benzoyl-3-n-pentylindole, respectively (Fig. 1) [10-12]. In many cases, the isomers endow different scheduling status. For instance, THJ-2201 is scheduled in the US, Japan, and some European countries; however, FUBIMINA, while being scheduled in Japan since August 2014, is currently available over the Internet in the US. Therefore, it is essential to confirm which SC is consumed for the purpose of legal prosecution. Previously, DeRuiter et al. [12] described the differentiation of 1-npentyl-3-benzoylindole and 1-benzoyl-3-n-pentylindole. However, the methods are limited to the parent drugs without considering metabolites. Because all previously studied SCs are extensively metabolized, major metabolites information is more important than parents for confirmation of intake.

FUBIMINA [(1-(5-fluoropentyl)-1H-benzo[d]imidazol2-yl)(naphthalen-1-yl)methanone] was first detected in 2013 by Uchiyama et al. [11] in herbal-type products purchased on the Internet. FUBIMINA, a potent agonist for the $\mathrm{CB}_{2}$ receptor $\left(K_{i}=23.45 \mathrm{nM}\right)$ [3], and THJ-2201 are fluoro derivatives of BIM-018 and THJ-018, respectively

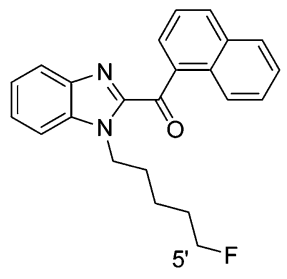

FUBIMINA (BIM-2201)

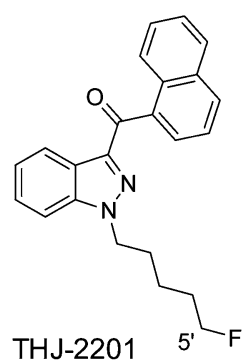

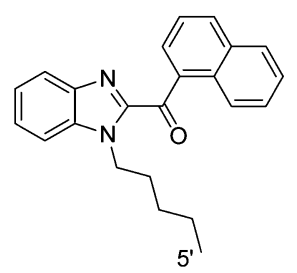

BIM-018

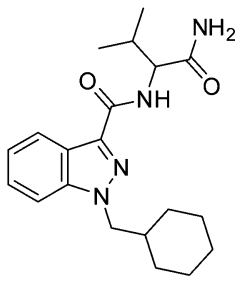

AB-CHMINACA

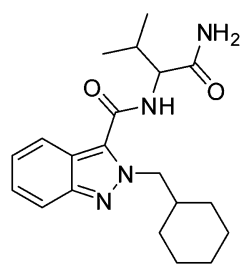

AB-CHMINACA $2 \mathrm{H}$-indazole analog

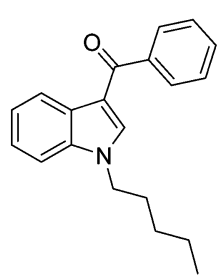

1-n-Pentyl-3-benzoylindole

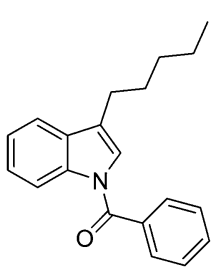

1-Benzoyl-3-n-pentylindole

Fig. 1 Structures of pairs of isomeric synthetic cannabinoids 
analysis is time-consuming and less reliable due to the uncertainty of fragment elemental composition.

Our aims were to characterize human FUBIMINA metabolism with human hepatocytes, recommend appropriate metabolites for clinical screening, and propose strategies to distinguish FUBIMINA from THJ-2201 intake. Human hepatocytes contain all required cofactors, enzymes, and an intact membrane, producing ratios of metabolites more closely matched to those in authentic human urine than those produced from human liver microsomes (HLM) [20-22]. Our approach using HLM to determine parent compound half-life and properly design human hepatocyte experiments, human hepatocyte incubation to produce phase I and phase II metabolites followed by HR-MS analysis, and sophisticated data analysis have proved successful in predicting major human urine metabolites for other SCs including AB-PINACA [23], AB-FUBINACA [24], FDU-PB-22, and FUB-PB-22 [14]. The spectral data for major metabolites will be highly useful for clinical scientists to incorporate into their urine screening methods to identify FUBIMINA intake.

\section{Materials and methods}

\section{Chemicals and reagents}

FUBIMINA (purity $98.42 \%$ ) was verified and kindly provided by the Drug Enforcement Administration, Department of Justice, USA. THJ-2201 and NADPH were purchased from Cayman Chemical (Ann Arbor, MI, USA). Cryopreserved human hepatocytes (10-donor pool), GRO $\mathrm{CP}$ and GRO KHB (Krebs-Henseleit Buffer) buffer for hepatocytes, and HLM (50-donor pool) were acquired from BioreclamationIVT (Baltimore, MD, USA). Acetonitrile was purchased from Sigma-Aldrich (St. Louis, MO, USA). LC-MS grade water and formic acid were purchased from Fisher Scientific (Waltham, MA, USA).

\section{Metabolic stability of FUBIMINA in human liver microsomes}

The HLM metabolic stability assays were performed in the same manner as in our previous manuscripts [25, 26]. Final concentration of FUBIMINA in HLM incubation system was $1 \mu \mathrm{M}$. Samples were centrifuged at $15,000 \mathrm{~g}\left(4{ }^{\circ} \mathrm{C}\right.$, 5 min); supernatant was stored at $-80{ }^{\circ} \mathrm{C}$ until analysis. When analyzing, the samples were thawed, vortexed, and centrifuged again. Then, $10 \mu \mathrm{L}$ supernatant was diluted in $990 \mu \mathrm{L}$ mobile phase A/B (90: 10, v/v). Ten microliters was injected to determine remaining FUBIMINA.

The high-performance liquid chromatography (HPLC) system consisted of two LC-20ADxr pumps, a DGU-
20A3R degasser, a SIL-20ACxr autosampler, and a CTO20A column oven (Shimadzu, Columbia, MD, USA). The Kinetex $\mathrm{C}_{18}$ column $(100 \mathrm{~mm} \times 2.1 \mathrm{~mm}, 2.6 \mu \mathrm{m}$; Phenomenex, Torrance, CA, USA) was fitted with a KrudKatcher Ultra HPLC in-line filter $(0.5 \mu \mathrm{m} \times 0.1 \mathrm{~mm}$, Phenomenex). Mobile phases were $0.1 \%$ formic acid in water (A) and $0.1 \%$ formic acid in acetonitrile (B). The gradient elution started at $10 \% \mathrm{~B}$ for $0.5 \mathrm{~min}$, ramped to $95 \% \mathrm{~B}$ at $10 \mathrm{~min}$, then held until $12.5 \mathrm{~min}$ before reequilibrating at $10 \% \mathrm{~B}$ for $2.5 \mathrm{~min}$. Total run time was $15 \mathrm{~min}$ with a flow rate of $0.3 \mathrm{~mL} / \mathrm{min}$. Column and autosampler temperatures were 40 and $4{ }^{\circ} \mathrm{C}$, respectively.

Data acquisition was performed on 3200 QTRAP mass spectrometer (Sciex, Redwood City, CA, USA) with Analyst software (version 1.6) in positive electrospray ionization (+ESI) mode. Ion source parameters were as follows: source temperature $500{ }^{\circ} \mathrm{C}$; ion spray voltage, $4000 \mathrm{~V}$; curtain gas, 30 psi; gas 1, 45 psi; gas 2, 70 psi. Two ion transitions were monitored for FUBIMINA $(\mathrm{m} / \mathrm{z}$ $361 \rightarrow 155 ; \mathrm{m} / \mathrm{z} 361 \rightarrow 177)$. Mass parameters were as follows: declustering potential, $61 \mathrm{~V}$; collision energy, $43 \mathrm{eV}(\mathrm{m} / \mathrm{z} 361 \rightarrow 155)$ and $35 \mathrm{eV}(\mathrm{m} / z 361 \rightarrow 177)$.

FUBIMINA peak areas were plotted against time, and in vitro microsomal half-life $\left(T_{1 / 2}\right)$ and intrinsic clearance $\left(\mathrm{CL}_{\text {int, micr }}\right)$ were calculated. Microsomal intrinsic clearance was then scaled to whole-liver dimensions, yielding intrinsic clearance $\left(\mathrm{CL}_{\mathrm{int}}\right)$. Human hepatic clearance $\left(\mathrm{CL}_{\mathrm{H}}\right)$ and extraction ratio (ER) were predicated based on $\mathrm{CL}_{\mathrm{int}}$ without considering plasma protein binding.

\section{Metabolite identification in human hepatocytes}

Hepatocyte incubation was carried out as previously described [15, 25, 26]. Cryopreserved human hepatocytes were washed twice with GRO CP medium and GRO KHB buffer. Cell viability was evaluated with Trypan blue $(0.4 \%, v / v)$ exclusion method, assuring greater than $80 \%$ viability. FUBIMINA was incubated at a final concentration of $10 \mu \mathrm{M}$ with human hepatocytes $\left(1 \times 10^{6}\right.$ cells $\left./ \mathrm{mL}\right)$ in a 24-well plate (BD Biosciences, San Jose, CA, USA). Final incubation volume was $500 \mu \mathrm{L}$ and samples were incubated at $37^{\circ} \mathrm{C}$. Chemical stability of FUBIMINA in the KHB buffer, and hepatocyte incubation without FUBIMINA also were performed $\left(37{ }^{\circ} \mathrm{C}, 3 \mathrm{~h}\right)$ to determine whether metabolites are dependent on hepatocytes enzymes or are impurities from hepatocyte itself. Diclofenac was incubated as a positive control to ensure metabolic activity of the hepatocytes by monitoring formation of $4^{\prime}$-hydroxydiclofenac and acyl $\beta$-D-glucuronide diclofenac. The reactions were quenched by adding $500 \mu \mathrm{L}$ ice-cold acetonitrile at 0 and $3 \mathrm{~h}$ incubation. Samples were stored at $-80{ }^{\circ} \mathrm{C}$ until analysis. Before analysis, samples were thawed, vortexed thoroughly, and $100 \mu \mathrm{L}$ acetonitrile 
added to $100 \mu \mathrm{L}$ samples. After vortexing and centrifugation at $15,000 \mathrm{~g}\left(4^{\circ} \mathrm{C}, 5 \mathrm{~min}\right)$, supernatant was transferred to a new $10-\mathrm{mL}$ plastic tube, evaporated to dryness under nitrogen at $40{ }^{\circ} \mathrm{C}$, and reconstituted in $150 \mu \mathrm{L}$ mobile phase A/B $(80: 20, v / v)$. Fifteen microliters of the reconstituted solution was injected for metabolite identification.

The HPLC system consisted of two LC-20ADxr pumps, a DGU-20A5R degasser, a SIL-20ACxr autosampler, and a CTO-20 AC column oven (Shimadzu). Chromatographic separation was achieved on an Ultra Biphenyl column $(100 \times 2.1 \mathrm{~mm}, 3 \mu \mathrm{m}$; Restek, Bellefonte, PA, USA $)$ with a guard column containing the same packing material. Gradient elution was performed with $0.1 \%$ formic acid in water (A) and $0.1 \%$ formic acid in acetonitrile (B) at a flow rate of $0.5 \mathrm{~mL} / \mathrm{min}$. Initial gradient conditions were $20 \% \mathrm{~B}$, held for $0.5 \mathrm{~min}$; then increased to $95 \% \mathrm{~B}$ over $10.5 \mathrm{~min}$, held until $13.0 \mathrm{~min}$; and returned to $20 \% \mathrm{~B}$ at $13.1 \mathrm{~min}$ and held until $15.0 \mathrm{~min}$. The column and autosampler were maintained at 30 and $4{ }^{\circ} \mathrm{C}$, respectively.

Data were acquired on a $5600^{+}$TripleTOF MS (Sciex) in +ESI mode. MS data were acquired by informationdependent acquisition (IDA) in combination with multiple mass defect filters (MDF) and dynamic background subtraction (DBS). Ion source parameters were as follows: curtain gas, $45 \mathrm{psi}$; gas 1, $60 \mathrm{psi}$; gas $2,75 \mathrm{psi}$; source temperature $650^{\circ} \mathrm{C}$; ion spray voltage, $4000 \mathrm{~V}$; declustering potential, $80 \mathrm{~V}$; entrance potential, $10 \mathrm{~V}$. For IDA, spectra exceeding $100 \mathrm{cps}$ were selected for the metabolism-dependent tandem mass spectrometry MS/MS scan, isotopes within $1.5 \mathrm{Da}$ were excluded, mass tolerance was $50 \mathrm{mDa}$, and collision energy was set to $35 \pm 15 \mathrm{eV}$. The MS was calibrated automatically every three injections.

MetabolitePilot (version 1.5, Sciex) characterized metabolites with different peak-finding algorithms including MDF, predicted biotransformation, generic liquid chromatography (LC) peak finding, common product ion and neutral loss; LC peak intensity threshold was $500 \mathrm{cps}$, MS $200 \mathrm{cps}$, and MS/MS $50 \mathrm{cps}$. Special attention was given to phase II metabolites that were susceptible to insource fragmentation.

To distinguish FUBIMINA from THJ-2201, THJ-2201 hepatocyte samples incubated in the same procedure were analyzed with the same acquisition and processing methods.

\section{Results and discussion}

\section{Metabolic stability evaluation in human liver microsomes}

After determining remaining FUBIMINA at different time points, the in vitro $T_{1 / 2}$ was calculated to be $4.9 \pm 0.03 \mathrm{~min}$, and in vitro $\mathrm{CL}_{\mathrm{int}}$, micr was $0.142 \mathrm{~mL} / \mathrm{min} / \mathrm{mg}$, corresponding to intrinsic clearance $\left(\mathrm{CL}_{\mathrm{int}}\right)$ of $134.1 \mathrm{~mL} / \mathrm{min} / \mathrm{kg}$ after scaling to whole-liver dimensions [27]. Without considering plasma protein binding and with a simplified Rowland's equation $[21,28]$, we calculated the predicted human $\mathrm{CL}_{\mathrm{H}}$ as $17.4 \mathrm{~mL} / \mathrm{min} / \mathrm{kg}$ and ER as 0.87 .

Metabolic stability, expressed as in vitro $T_{1 / 2}$ and $\mathrm{CL}_{\mathrm{int}}$ defines a drug's susceptibility to metabolism. These values facilitate the prediction of in vivo hepatic clearance, in vivo $T_{1 / 2}$, and bioavailability [21, 29]. According to criteria proposed by Lave et al. [30], short $T_{1 / 2}$, large $\mathrm{CL}_{\text {int }}$, and high ER demonstrate that FUBIMINA is a high clearance drug.

\section{Fragmentation of FUBIMINA and THJ-2201}

FUBIMINA $\left([\mathrm{M}+\mathrm{H}]^{+}, m / z, 361.1730\right)$ eluted at $8.70 \mathrm{~min}$ and displayed characteristic product ions at $\mathrm{m} / \mathrm{z}, 273.1041$, 233.1099, 213.1036, 177.0467, 155.0499, 145.0408, and 127.0552 (Fig. 2a, c). Fragment $\mathrm{m} / \mathrm{z} 273.1041$ was generated via neutral loss of the fluoropentyl chain; $\mathrm{m} / \mathrm{z}$ 233.1099 and 145.0408 represent the benzimidazole core with and without the fluoropentyl chain. Neutral HF loss from $\mathrm{m} / \mathrm{z} 233.1099$ yielded $\mathrm{m} / \mathrm{z}, 213.1036 ; \mathrm{m} / \mathrm{z} 177.0467$ is formed by fluorine rearrangement, and $m / z, 155.0499$ and 127.0552 correspond to the naphthalene moiety with or without the acyl group.

THJ-2201 eluted later at $9.21 \mathrm{~min}$ (Fig. 2b), and produced similar fragments (Fig. 2d), but with quite different relative ion abundances, as for $m / z 177.0467$ and 155.0499. Notably, the prominent fragment $m / z 273.1041$ in FUBIMINA was not observed in THJ-2201.

\section{FUBIMINA metabolites in human hepatocytes}

In the $3 \mathrm{~h}$ hepatocyte sample, 35 metabolites were detected (Fig. 3; Table 1). No metabolites were observed following FUBIMINA incubation in buffer for $3 \mathrm{~h}$, indicating that metabolite formation was enzyme-dependent. An overview of the metabolic pathway is shown in Fig. 4. Metabolites were labeled " $M$ " in sequential eluting order. Table 1 lists metabolites with pathway description, retention time, $\mathrm{m} / \mathrm{z}$, mass error, formula, fragment ions, and peak areas. Based on MS peak areas, primary metabolites were BIM-018 pentanoic acid (M33), 5'-OH-BIM-018 glucuronide (M20), and BIM-018 pentanoic acid dihydrodiol (M7). The top ten major metabolites were elucidated as described below, with product ion spectra shown in Fig. 5.

Oxidative defluorination and further oxidation plus glucuronidation

For M34, precursor $\mathrm{m} / \mathrm{z} 359.1767$ and fragment $\mathrm{m} / \mathrm{z}$ 231.1125 were $1.9963 \mathrm{Da}(-\mathrm{F}+\mathrm{OH})$ less than the corresponding ions of FUBIMINA (Fig. 6e), indicating fluorine 
Fig. 2 Extracted ion chromatograms of FUBIMINA $(\mathrm{m} / \mathrm{z}$ 361.0730, a) and THJ-2201 $(\mathrm{m} / \mathrm{z}$ 361.0730, b), their product ion spectra and proposed fragmentation (FUBIMINA, c; THJ-2201, d)
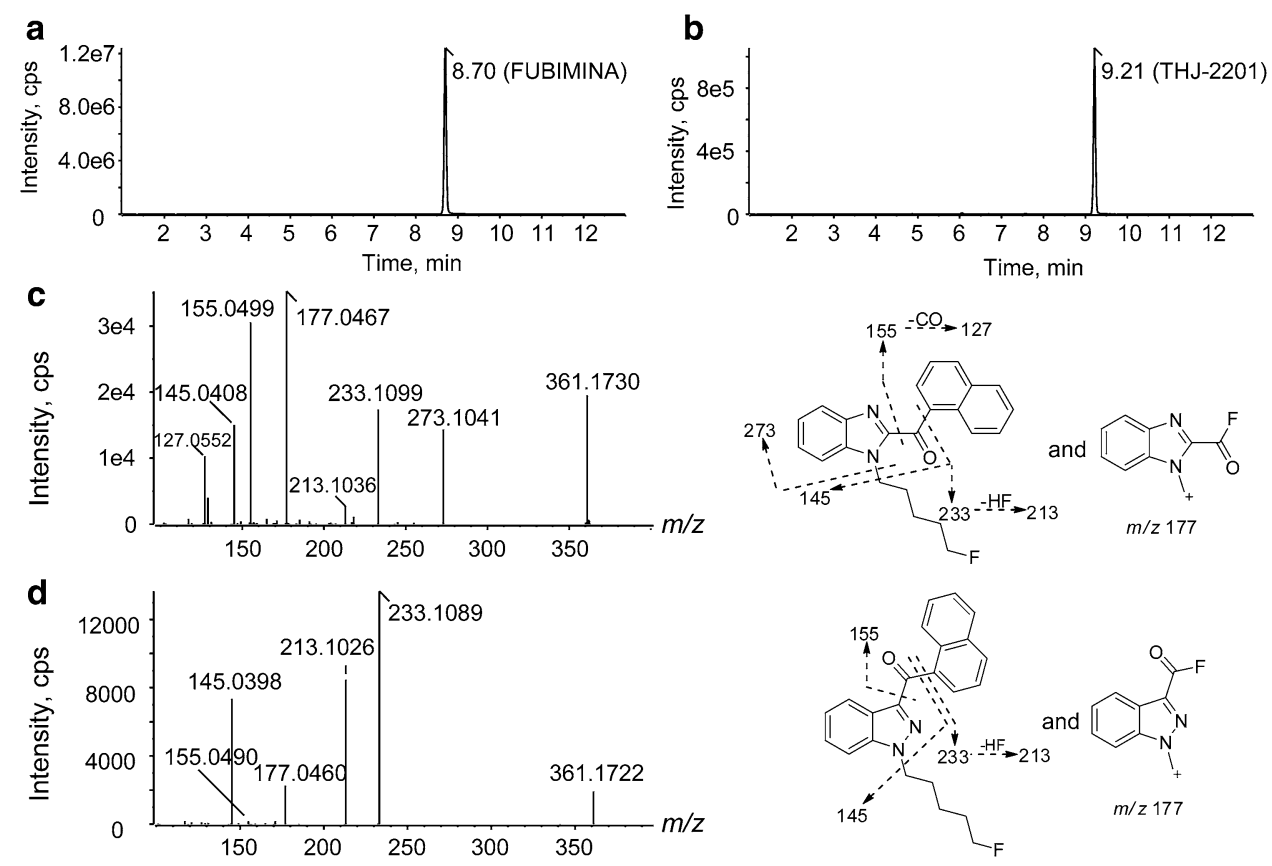

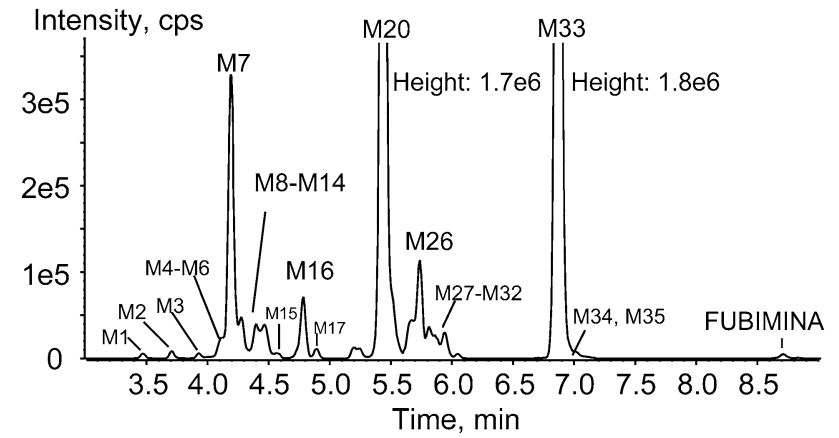

Fig. 3 Metabolic profile of FUBIMINA after $3 \mathrm{~h}$ incubation in human hepatocytes

replacement by a hydroxyl group. Other fragments $\mathrm{m} / \mathrm{z}$ 213.1019, 155.0491, 145.0396, and 127.0542 were the same as for FUBIMINA, indicating that the benzimidazole and naphthalene moieties were unmodified. Therefore, M34 was proposed as the oxidative defluorination metabolite, i.e., 5'-OH-BIM-018. Oxidative defluorination also was the primary metabolic pathway of THJ-2201 [15]. M34 was easily glucuronidated to yield 5'-OH-BIM-018 glucuronide (M20) (Fig. 5a), the second most abundant FUBIMINA metabolite. M20 fragment $\mathrm{m} / \mathrm{z} 359.1765$ was formed via neutral glucuronide loss $(-176.0324 \mathrm{Da})$. Other fragments, $\mathrm{m} / \mathrm{z}$ 273.1026, 231.1127, 155.0485, and 145.0395, were the same as for M34.

The predominant M34 metabolic pathway was oxidation to BIM-018 pentanoic acid (M33, Fig. 6g). M33 generated fragments at $\mathrm{m} / \mathrm{z} 273.1038,245.0930,201.1032,155.0500$, 145.0404, and 127.0549. Among them, $\mathrm{m} / \mathrm{z}$ 273.1038, 155.0500, 145.0404, and 127.0549 also were present in FUBIMINA, indicating naphthalene and benzimidazole moieties were intact. The $\mathrm{m} / \mathrm{z}, 201.1032$ was formed via neutral $\mathrm{CO}_{2}$ loss (-43.9898 Da) from $\mathrm{m} / \mathrm{z}$ 245.0930. Therefore, M33 was tentatively assigned as BIM-018 pentanoic acid. Subsequent glucuronidation of M33 led to M18/M19/M22 that shared similar product ion spectra, all of them displaying neutral loss of glucuronide (Fig. 5d). M18/M19/M22 appeared in a cluster probably because of acyl glucuronide migration [31].

M34 also underwent benzimidazole hydroxylation and glucuronidation to M14/M16. Their major fragment, $\mathrm{m} / \mathrm{z}$ 375.1714 was generated via loss of glucuronide (Fig. 5c). Fragments $m / z, 289.0979$ and 161.0343 were $15.9947 \mathrm{Da}$ $(+\mathrm{O})$ larger than corresponding M34 ions, suggesting benzimidazole hydroxylation. Glucuronidation may also occur on the benzimidazole hydroxyl group.

M34 also underwent dihydrodiol formation yielding M10. For M10 (Fig. 5f), fragments $\mathrm{m} / \mathrm{z} 307.1081$ and 179.0446 were $34.0051 \mathrm{Da}(+2 \mathrm{O}+2 \mathrm{H})$ larger than M34 corresponding ions, indicating dihydrodiol formation on benzimidazole. Dihydrodiol ions lost water easily within the MS collision cell, generating ions at $m / z 289.0957$ and 161.0345. Similarly, M33 (BIM-018 pentanoic acid) produced a dihydrodiol metabolite (M7), the 3rd largest metabolite. M7 shared fragments $\mathrm{m} / \mathrm{z}, 245.0914,201.1023$, and 145.0395 with M33 (Fig. 6i); fragments $m / z, 307.1077$ 
Table 1 Identification of FUBIMINA metabolites after $3 \mathrm{~h}$ incubation with human hepatocytes. Fragment ions were expressed in nominal mass because of space restraint

\begin{tabular}{|c|c|c|c|c|c|c|c|c|}
\hline ID & Metabolic pathway & $\begin{array}{l}\text { Time } \\
(\min )\end{array}$ & $\begin{array}{l}{[\mathrm{M}+\mathrm{H}]^{+}} \\
(\mathrm{m} / z)\end{array}$ & $\begin{array}{l}\text { Mass } \\
\text { error } \\
(\mathrm{ppm})\end{array}$ & Formula & Fragment ions & Area & Rank \\
\hline & Parent & 8.72 & 361.1711 & 0.1 & $\mathrm{C}_{23} \mathrm{H}_{21} \mathrm{~N}_{2} \mathrm{OF}$ & $\begin{array}{l}273,233,213, \\
177,155,145, \\
127\end{array}$ & $2.48 \mathrm{E}+04$ & NA \\
\hline M1 & M34 + dioxidation + glucuronidation & 3.47 & 567.1984 & 1.8 & $\mathrm{C}_{29} \mathrm{H}_{30} \mathrm{~N}_{2} \mathrm{O}_{10}$ & $\begin{array}{l}391,305,247 \\
229,171,161\end{array}$ & $1.83 \mathrm{E}+04$ & 29 \\
\hline M2 & M33 + oxidation & 3.71 & 389.1505 & 2.3 & $\mathrm{C}_{23} \mathrm{H}_{20} \mathrm{~N}_{2} \mathrm{O}_{4}$ & $\begin{array}{c}371,289,201 \\
171,145\end{array}$ & $2.59 \mathrm{E}+04$ & 27 \\
\hline M3 & M34 + dioxidation + glucuronidation & 3.92 & 567.1973 & 0.0 & $\mathrm{C}_{29} \mathrm{H}_{30} \mathrm{~N}_{2} \mathrm{O}_{10}$ & $\begin{array}{l}391,247,171 \\
161\end{array}$ & $2.12 \mathrm{E}+04$ & 28 \\
\hline M4 & $N$-Dealkylation + oxidation + glucuronidation & 4.09 & 465.1298 & 1.2 & $\mathrm{C}_{24} \mathrm{H}_{20} \mathrm{~N}_{2} \mathrm{O}_{8}$ & $289,161,155$ & $1.22 \mathrm{E}+04$ & 34 \\
\hline M5 & Oxidation + dihydrodiol & 4.10 & 411.1720 & 1.4 & $\mathrm{C}_{23} \mathrm{H}_{23} \mathrm{~N}_{2} \mathrm{O}_{4} \mathrm{~F}$ & $\begin{array}{l}393,375,289, \\
271,189,171 \\
145\end{array}$ & $4.27 \mathrm{E}+04$ & 20 \\
\hline M6 & M34 + dihydrodiol & 4.14 & 393.1825 & 4.0 & $\mathrm{C}_{23} \mathrm{H}_{24} \mathrm{~N}_{2} \mathrm{O}_{4}$ & $\begin{array}{l}375,289,271 \\
189,171,145\end{array}$ & $4.20 \mathrm{E}+04$ & 21 \\
\hline M7 & M33 + dihydrodiol & 4.19 & 407.1613 & 2.9 & $\mathrm{C}_{23} \mathrm{H}_{22} \mathrm{~N}_{2} \mathrm{O}_{5}$ & $\begin{array}{l}389,371,307 \\
289,271,245, \\
201,189,171, \\
145\end{array}$ & $9.27 \mathrm{E}+05$ & 3 \\
\hline M8 & M34 + oxidation + glucuronidation & 4.21 & 551.2030 & 1.2 & $\mathrm{C}_{29} \mathrm{H}_{30} \mathrm{~N}_{2} \mathrm{O}_{9}$ & $\begin{array}{c}375,289,231 \\
171,145\end{array}$ & $1.07 \mathrm{E}+05$ & 11 \\
\hline M9 & Dioxidation + glucurnidation & 4.23 & 569.1932 & 0.4 & $\mathrm{C}_{29} \mathrm{H}_{29} \mathrm{~N}_{2} \mathrm{O}_{9} \mathrm{~F}$ & $\begin{array}{c}393,289,249 \\
171,145\end{array}$ & $6.40 \mathrm{E}+04$ & 17 \\
\hline M10 & M34 + dihydrodiol & 4.28 & 393.1823 & 3.5 & $\mathrm{C}_{23} \mathrm{H}_{24} \mathrm{~N}_{2} \mathrm{O}_{4}$ & $\begin{array}{l}307,289,265 \\
179,161,155\end{array}$ & $1.10 \mathrm{E}+05$ & 9 \\
\hline M11 & M34 + dioxidation + glucuronidation & 4.36 & 567.1978 & 0.9 & $\mathrm{C}_{29} \mathrm{H}_{30} \mathrm{~N}_{2} \mathrm{O}_{10}$ & $391,305,231$ & $1.40 \mathrm{E}+04$ & 32 \\
\hline M12 & M33 + oxidation & 4.40 & 389.1506 & 2.6 & $\mathrm{C}_{23} \mathrm{H}_{20} \mathrm{~N}_{2} \mathrm{O}_{4}$ & $\begin{array}{l}289,245,201 \\
171,145\end{array}$ & $9.65 \mathrm{E}+04$ & 12 \\
\hline M13 & Oxidation + dihydrodiol & 4.40 & 411.1711 & -0.8 & $\mathrm{C}_{23} \mathrm{H}_{23} \mathrm{~N}_{2} \mathrm{O}_{4} \mathrm{~F}$ & $\begin{array}{c}393,307,289 \\
161,155\end{array}$ & $3.51 \mathrm{E}+04$ & 24 \\
\hline M14 & M34 + oxidation + glucuronidation & 4.47 & 551.2033 & 1.5 & $\mathrm{C}_{29} \mathrm{H}_{30} \mathrm{~N}_{2} \mathrm{O}_{9}$ & $\begin{array}{l}375,289,247 \\
161\end{array}$ & $1.52 \mathrm{E}+05$ & 7 \\
\hline M15 & M34 + dioxidation + glucuronidation & 4.57 & 567.1973 & 0.0 & $\mathrm{C}_{29} \mathrm{H}_{30} \mathrm{~N}_{2} \mathrm{O}_{10}$ & 391,305 & $1.75 \mathrm{E}+04$ & 30 \\
\hline M16 & M34 + oxidation + glucuronidation & 4.78 & 551.2033 & 1.6 & $\mathrm{C}_{29} \mathrm{H}_{30} \mathrm{~N}_{2} \mathrm{O}_{9}$ & $\begin{array}{c}375,289,247 \\
161,155\end{array}$ & $2.37 \mathrm{E}+05$ & 5 \\
\hline M17 & Dioxidation + glucuronidation & 4.89 & 569.1935 & 0.9 & $\mathrm{C}_{29} \mathrm{H}_{29} \mathrm{~N}_{2} \mathrm{O}_{9} \mathrm{~F}$ & $\begin{array}{c}393,289,249 \\
193,161\end{array}$ & $3.58 \mathrm{E}+04$ & 23 \\
\hline M18 & M33 + glucuronidation & 5.20 & 549.1876 & 1.5 & $\mathrm{C}_{29} \mathrm{H}_{28} \mathrm{~N}_{2} \mathrm{O}_{9}$ & $\begin{array}{r}531,373,355 \\
273,201,155\end{array}$ & $6.98 \mathrm{E}+04$ & 14 \\
\hline M19 & M33 + glucuronidation & 5.39 & 549.1876 & 1.6 & $\mathrm{C}_{29} \mathrm{H}_{28} \mathrm{~N}_{2} \mathrm{O}_{9}$ & $\begin{array}{l}531,373,355 \\
273,201,155\end{array}$ & $6.59 \mathrm{E}+04$ & 16 \\
\hline M20 & M34 + glucuronidation & 5.43 & 535.2084 & 1.7 & $\mathrm{C}_{29} \mathrm{H}_{30} \mathrm{~N}_{2} \mathrm{O}_{8}$ & $\begin{array}{c}359,273,231 \\
155,145\end{array}$ & $4.53 \mathrm{E}+06$ & 2 \\
\hline M21 & Oxidation + glucuronidation & 5.45 & 553.1985 & 0.7 & $\mathrm{C}_{29} \mathrm{H}_{29} \mathrm{~N}_{2} \mathrm{O}_{8} \mathrm{~F}$ & $\begin{array}{l}377,273,249 \\
155\end{array}$ & $1.26 \mathrm{E}+05$ & 8 \\
\hline M22 & M33 + glucuronidation & 5.51 & 549.1876 & 1.5 & $\mathrm{C}_{29} \mathrm{H}_{28} \mathrm{~N}_{2} \mathrm{O}_{9}$ & $\begin{array}{l}531,373,355, \\
273,245,201, \\
155,145\end{array}$ & $2.35 \mathrm{E}+05$ & 6 \\
\hline M23 & M33 + oxidation & 5.56 & 389.1500 & 1.1 & $\mathrm{C}_{23} \mathrm{H}_{20} \mathrm{~N}_{2} \mathrm{O}_{4}$ & $\begin{array}{c}289,261,217 \\
161,155\end{array}$ & $2.88 \mathrm{E}+04$ & 25 \\
\hline
\end{tabular}


Table 1 continued

\begin{tabular}{|c|c|c|c|c|c|c|c|c|}
\hline ID & Metabolic pathway & $\begin{array}{l}\text { Time } \\
(\min )\end{array}$ & $\begin{array}{l}{[\mathrm{M}+\mathrm{H}]^{+}} \\
(m / z)\end{array}$ & $\begin{array}{l}\text { Mass error } \\
(\mathrm{ppm})\end{array}$ & Formula & Fragment ions & Area & Rank \\
\hline M24 & M33 + oxidation & 5.66 & 389.1507 & 2.9 & $\mathrm{C}_{23} \mathrm{H}_{20} \mathrm{~N}_{2} \mathrm{O}_{4}$ & $289,201,171,145$ & $6.07 \mathrm{E}+04$ & 19 \\
\hline M25 & Dioxidation + glucuronidation & 5.67 & 569.1935 & 0.9 & $\mathrm{C}_{29} \mathrm{H}_{29} \mathrm{~N}_{2} \mathrm{O}_{9} \mathrm{~F}$ & $393,305,265,177$ & $6.28 \mathrm{E}+04$ & 18 \\
\hline M26 & $\mathrm{N}$-Oxide + glucuronidation & 5.74 & 553.1992 & 2.1 & $\mathrm{C}_{29} \mathrm{H}_{29} \mathrm{~N}_{2} \mathrm{O}_{8} \mathrm{~F}$ & $\begin{array}{l}377,289,249,233,177, \\
161\end{array}$ & $4.11 \mathrm{E}+05$ & 4 \\
\hline M27 & Dihydrodiol & 5.81 & 395.1773 & 2.0 & $\mathrm{C}_{23} \mathrm{H}_{23} \mathrm{~N}_{2} \mathrm{O}_{3} \mathrm{~F}$ & $307,289,267,161,155$ & $1.07 \mathrm{E}+05$ & 10 \\
\hline M28 & M33 + oxidation & 5.84 & 389.1505 & 2.3 & $\mathrm{C}_{23} \mathrm{H}_{20} \mathrm{~N}_{2} \mathrm{O}_{4}$ & $273,217,155,145,127$ & $2.70 \mathrm{E}+04$ & 26 \\
\hline M29 & $\mathrm{N}$-Oxide + glucuronidation & 5.86 & 553.1990 & 1.6 & $\mathrm{C}_{29} \mathrm{H}_{29} \mathrm{~N}_{2} \mathrm{O}_{8} \mathrm{~F}$ & $\begin{array}{l}377,289,249,207,193 \\
177,161,145\end{array}$ & $6.82 \mathrm{E}+04$ & 15 \\
\hline M30 & M33 + oxidation & 5.93 & 389.1502 & 1.5 & $\mathrm{C}_{23} \mathrm{H}_{20} \mathrm{~N}_{2} \mathrm{O}_{4}$ & $289,201,171,145$ & $8.81 \mathrm{E}+04$ & 13 \\
\hline M31 & M34 + oxidation & 5.96 & 375.1712 & 2.4 & $\mathrm{C}_{23} \mathrm{H}_{22} \mathrm{~N}_{2} \mathrm{O}_{3}$ & $289,231,171,145$ & $1.24 \mathrm{E}+04$ & 33 \\
\hline M32 & Oxidation + sulfation & 6.04 & 457.1236 & 1.8 & $\mathrm{C}_{23} \mathrm{H}_{21} \mathrm{~N}_{2} \mathrm{O}_{5} \mathrm{FS}$ & $377,249,193,161,155$ & $1.52 \mathrm{E}+04$ & 31 \\
\hline M33 & $\begin{array}{l}\text { Oxidative defluorination to } \\
\text { carboxylic acid }\end{array}$ & 6.87 & 373.1558 & 3.0 & $\mathrm{C}_{23} \mathrm{H}_{20} \mathrm{~N}_{2} \mathrm{O}_{3}$ & $\begin{array}{l}273,245,201,155,145 \\
127\end{array}$ & $5.62 \mathrm{E}+06$ & 1 \\
\hline M34 & Oxidative defluorination & 6.92 & 359.1756 & 0.6 & $\mathrm{C}_{23} \mathrm{H}_{22} \mathrm{~N}_{2} \mathrm{O}_{2}$ & $273,231,155,145,127$ & $3.87 \mathrm{E}+04$ & 22 \\
\hline M35 & Oxidation & 7.01 & 377.1661 & 0.4 & $\mathrm{C}_{23} \mathrm{H}_{21} \mathrm{~N}_{2} \mathrm{O}_{2}$ & $\begin{array}{l}273,249,231,155,145, \\
127\end{array}$ & $1.15 \mathrm{E}+04$ & 35 \\
\hline
\end{tabular}

NA not applicable

and 189.0544 were 34.0039 Da larger than corresponding ions of M33, suggesting naphthalene dihydrodiol formation. Additionally, FUBIMINA itself underwent dihydrodiol formation yielding M27 (Fig. 5g). It shared fragment $m / z$ 155.0496 with FUBIMINA; fragments $m / z \quad 307.1080$ and 267.1161 were 34.0039 Da larger than $m / z 273.1041$ and 233.1099 of FUBIMINA, respectively, suggesting dihydrodiol formation on the benzimidazole.

\section{Fluoropentyl chain hydroxylation and further conjugation}

Fluoropentyl chain hydroxylation led to M35. M35 shared fragments $m / z$ 273.1024, 155.0487, 145.0393, and 127.0543 with FUBIMINA. M35 fragment $m / z$ 249.1038 was $15.9939 \mathrm{Da}(+\mathrm{O})$ larger than $\mathrm{m} / z, 233.1099$ of FUBIMINA, indicating fluoropentyl chain hydroxylation. The exact position of hydroxylation cannot be determined by MS. Sequential glucuronidation generated M21 (Fig. 5e).

\section{$N$-Oxide and glucuronidation}

Similar to THJ-2201 [15], an unusual pathway for SC, i.e., $N$-oxide glucuronidation was observed for FUBIMINA generating M26 and M29. Their most intense fragment $\mathrm{m} / \mathrm{z}$ 377.1673 was generated via glucuronide loss (Fig. 5b). Both metabolites shared product ions $\mathrm{m} / \mathrm{z}$ 233.1088, 177.0454, 155.0485, and 145.0396 with FUBIMINA, suggesting naphthalene, benzimidazole, and fluoropentyl chain were unmodified. However, fragments $\mathrm{m} / \mathrm{z}, 289.0979$, 249.1029, and 161.0343 indicate benzimidazole oxidation. Therefore, we propose M26/M29 as $N$-oxide glucuronides rather than glucuronides of hydroxylated metabolites. Generally, neutral $\mathrm{H}_{2} \mathrm{O}$ loss, instead of $\mathrm{O}$, was observed between fragments of hydroxylated metabolites, whereas it is common for $\mathrm{N}$-oxides to lose $\mathrm{O}$ or an $\mathrm{OH}$ radical [32, 33]. This unusual biotransformation dominated metabolism of an antidepressant vortioxetine [32], and anticonvulsants GI265080 and GW273293 [34]. FUBIMINA $N$-oxide itself was not observed, probably because it was easily glucuronidated.

\section{THJ-2201 major metabolites under the same condition}

THJ-2201 underwent similar metabolism in human hepatocytes as FUBIMINA, including oxidative defluorination, further carboxylation or glucuronidation, hydroxylation on the fluoropentyl chain, and dihydrodiol formation on naphthalene. THJ-2201 primary metabolites were 5'-OHTHJ-018 (F26) and its glucuronide F18, THJ-018 pentanoic acid (F25), and hydroxylated THJ-2201 (F13); their product ion spectra and fragmentation are shown in Fig. 6f, h, j. THJ-2201 metabolite names were taken from our previous manuscript characterizing its metabolic profile [15]. THJ-2201 and FUBIMINA marker metabolites are shown in Fig. 6b, d; notably, there are two isomer pairs (M34 and F26, M33 and F25). 
Fig. 4 Tentative metabolic pathways of FUBIMINA in human hepatocytes
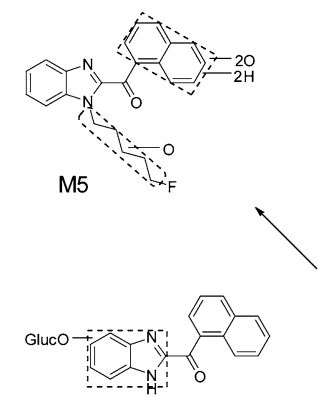

M4
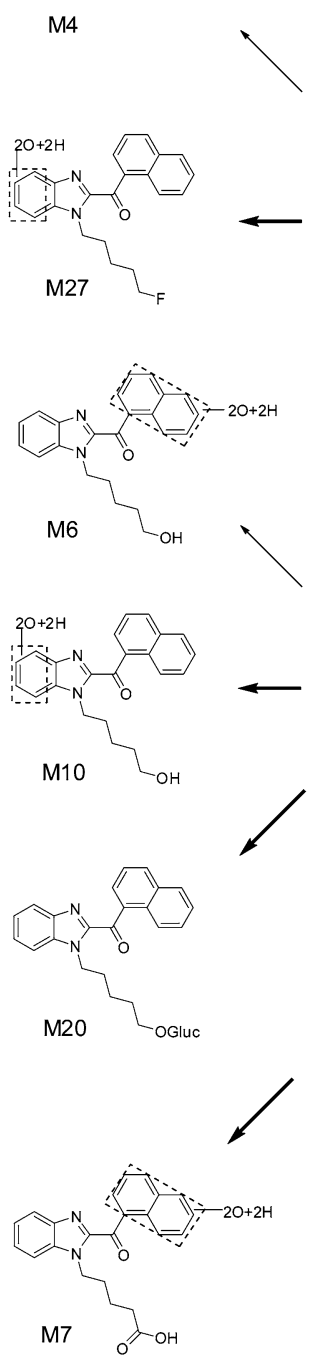
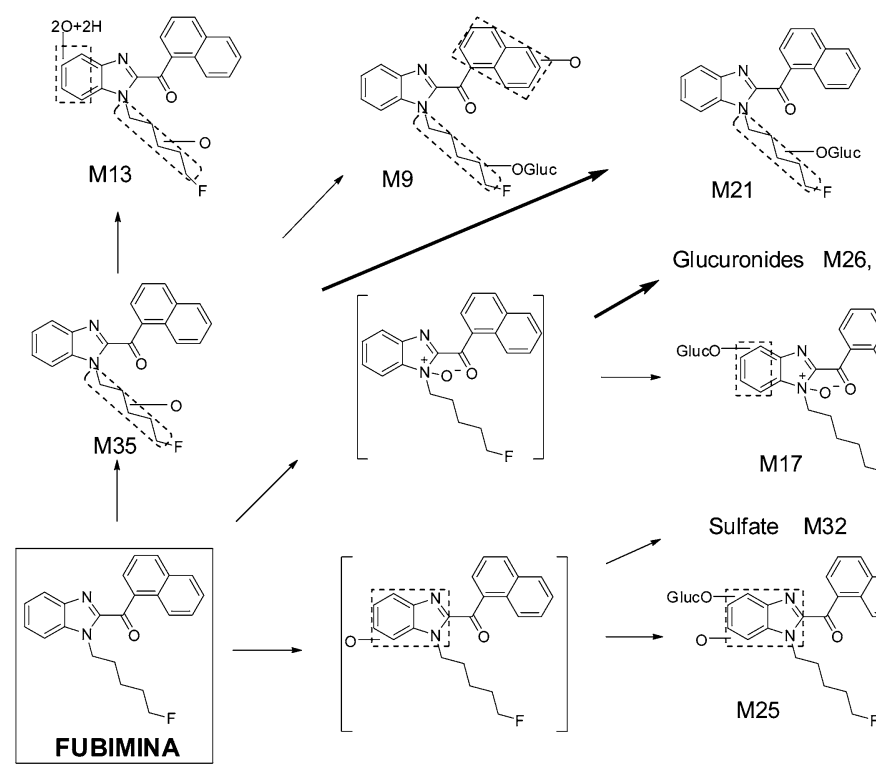

Glucuronides M26, M29
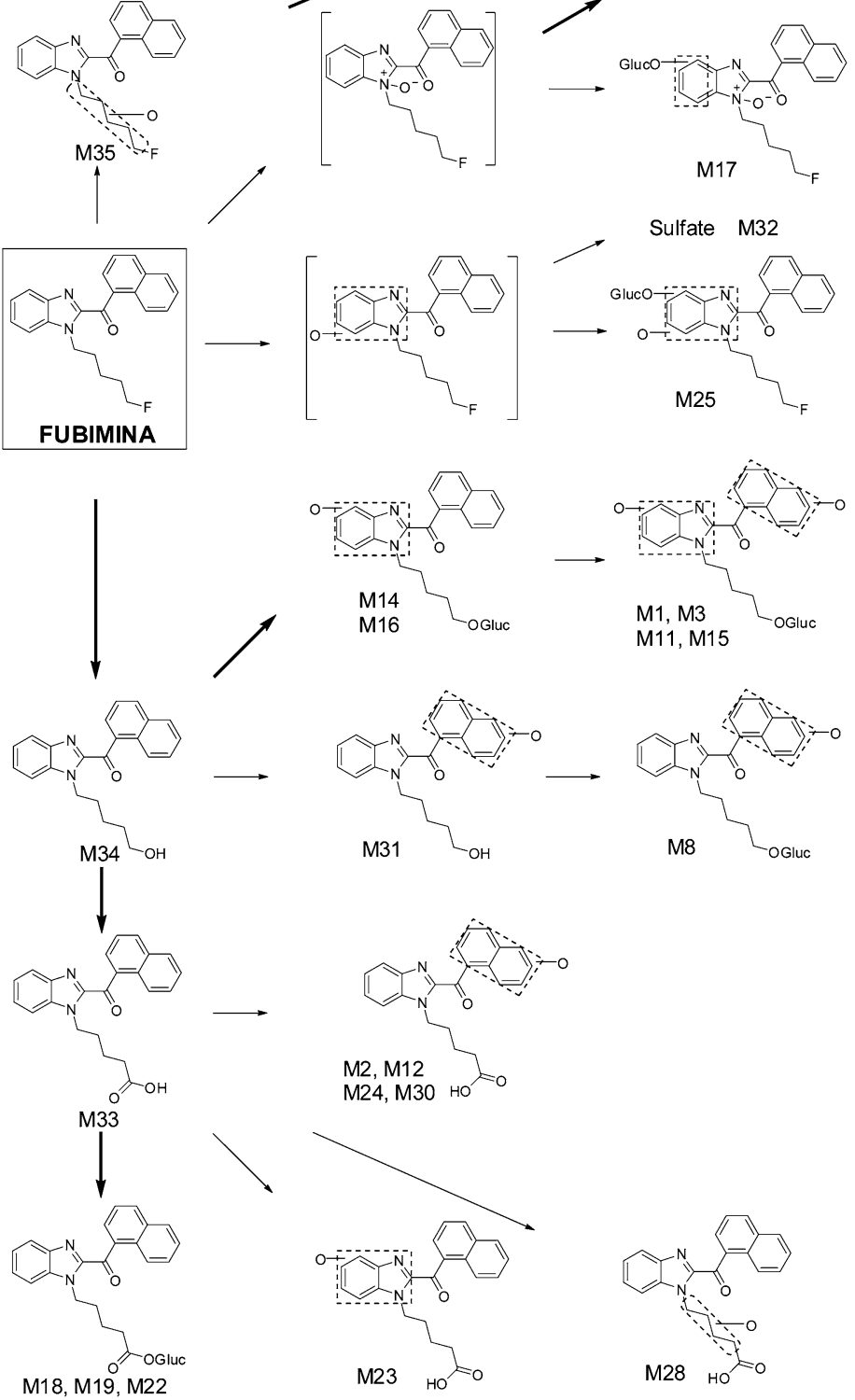

\section{Specific metabolites to distinguish FUBIMINA intake from THJ-2201}

Because FUBIMINA and THJ-2201 possess different legal status in different countries, unequivocal confirmation of which SC was taken is essential in forensic cases. In clinical and forensic urine screening tests, urine sam-

ples are usually hydrolyzed by $\beta$-glucuronidase [13, 35], and major phase I metabolites monitored. Additionally, glucuronide conjugates may degrade spontaneously at room temperature $[36,37]$. Therefore, we only propose phase I metabolites or aglycones of dominant phase II metabolites as urinary marker metabolites. This proved successful in documenting intake of AB-FUBINACA 

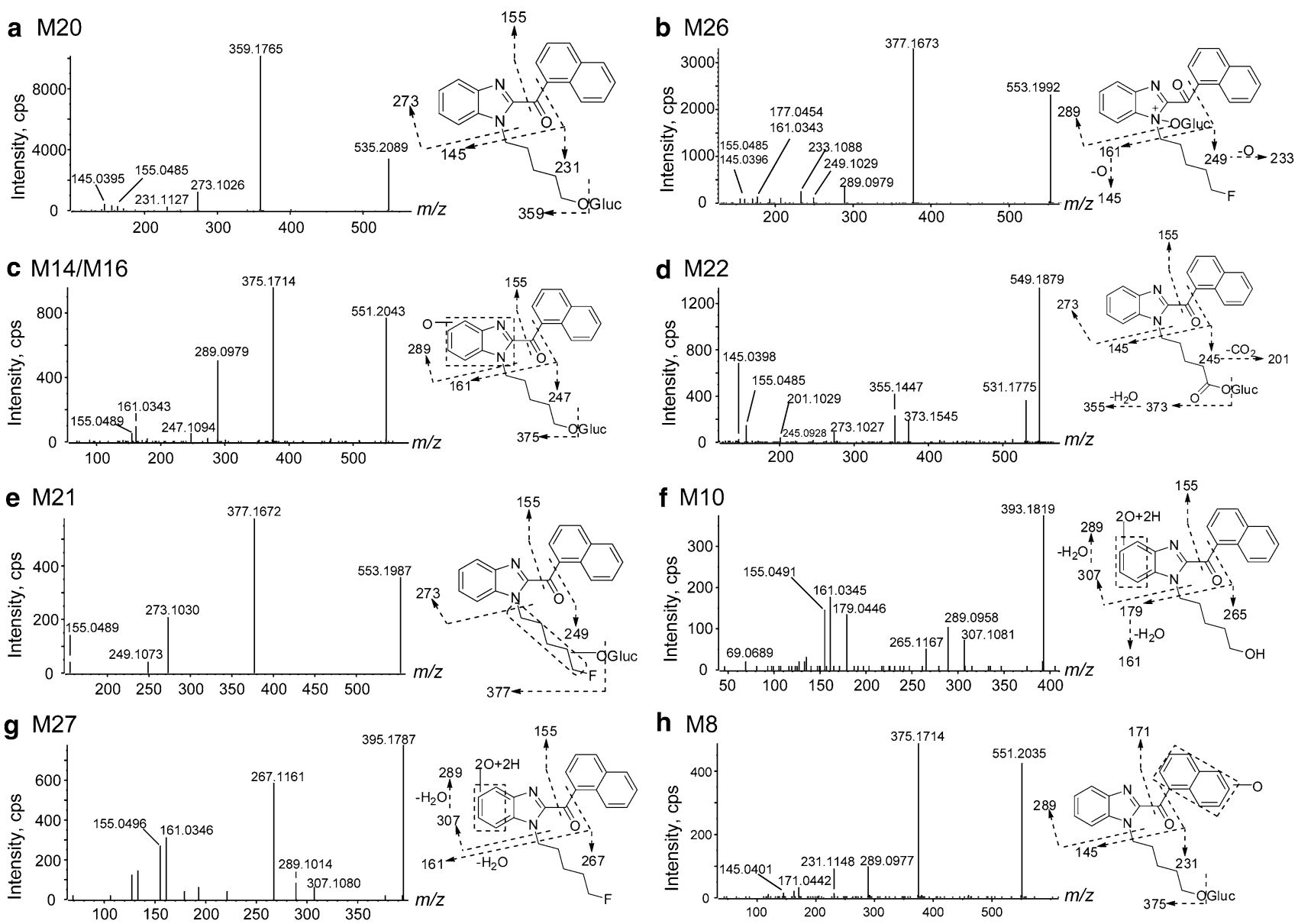

Fig. 5 FUBIMINA major metabolites, product ion spectra, proposed structures, and fragmentation patterns (M7 and M33 are shown in Fig. 6)

[24], AB-PINACA [23], FDU-PB-22 and FUB-PB-22 [14].

Based on our results, the most appropriate marker metabolites to document FUBIMINA intake (Fig. 6a, b) are $5^{\prime}$-OH-BIM-018 (M34), which is derived from the intense M20, BIM-018 pentanoic acid (M33), and BIM-018 pentanoic acid dihydrodiol (M7). For THJ-2201 (Fig. 6c, d), optimal marker metabolites include 5'-OH-THJ-018 (F26), THJ-018 pentanoic acid (F25), and hydroxylated THJ-2201 (F13). The isomers M34 and F26 as well as M33 and F25 elute at different retention times, i.e., 6.92 (M34) versus $7.57 \mathrm{~min}$ (F26) and 6.87 (M33) versus $7.49 \mathrm{~min}$ (F25) (Fig. 6a, c), and are distinguishable by different product ion spectra. A unique fragment, $\mathrm{m} / \mathrm{z} 273.1030$ was always a major fragment in FUBIMINA and its metabolites, whereas it was only a negligible ion in THJ-2201 and its metabolites. For instance, $m / z, 273.1030$ and 155.0491 were the dominant M34 fragments (Fig. 6e) whereas $\mathrm{m} / \mathrm{z}, 213.1026$ and 145.0400 were the top F26 fragments and $\mathrm{m} / z, 273.1030$ was not observed at all (Fig. 6f). In M33, $m / z 273.1038$ was the most intense fragment; but it is absent in F25. Additionally, presence of both $\mathrm{m} / \mathrm{z}, 245.0930$ and 201.1032 are characteristic for M33, versus $\mathrm{m} / \mathrm{z}$ 245.0930, 227.0817, and 217.0965 for F25 (Fig. 6g, h).

Urine is the most common routine matrix for drug screening because of easy accessibility and high drug concentration compared to blood or oral fluid. There are limitations to extrapolating human hepatocyte data to human urine. Extrahepatic metabolism [38], transporter involvement [39], and metabolite enrichment in urine [40] might change relative abundance of urinary metabolites, and thus selection of marker metabolites. Therefore, it is preferable to obtain FUBIMINA and THJ-2201 positive urine case samples, and confirm whether recommended hepatocyte marker metabolites match those present in authentic urine after SC intake. Unfortunately, such samples are currently unavailable despite our efforts to obtain them. 

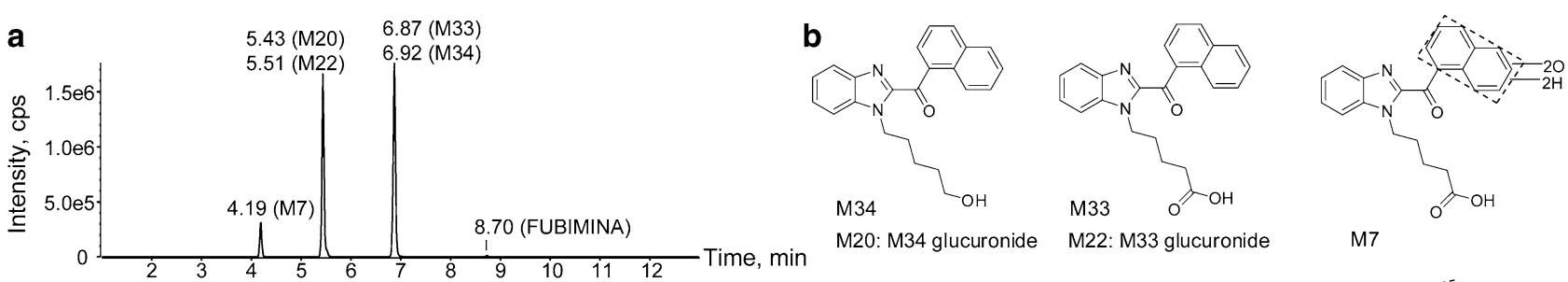

M20: M34 glucuronide

M22: M33 glucuronide

M7
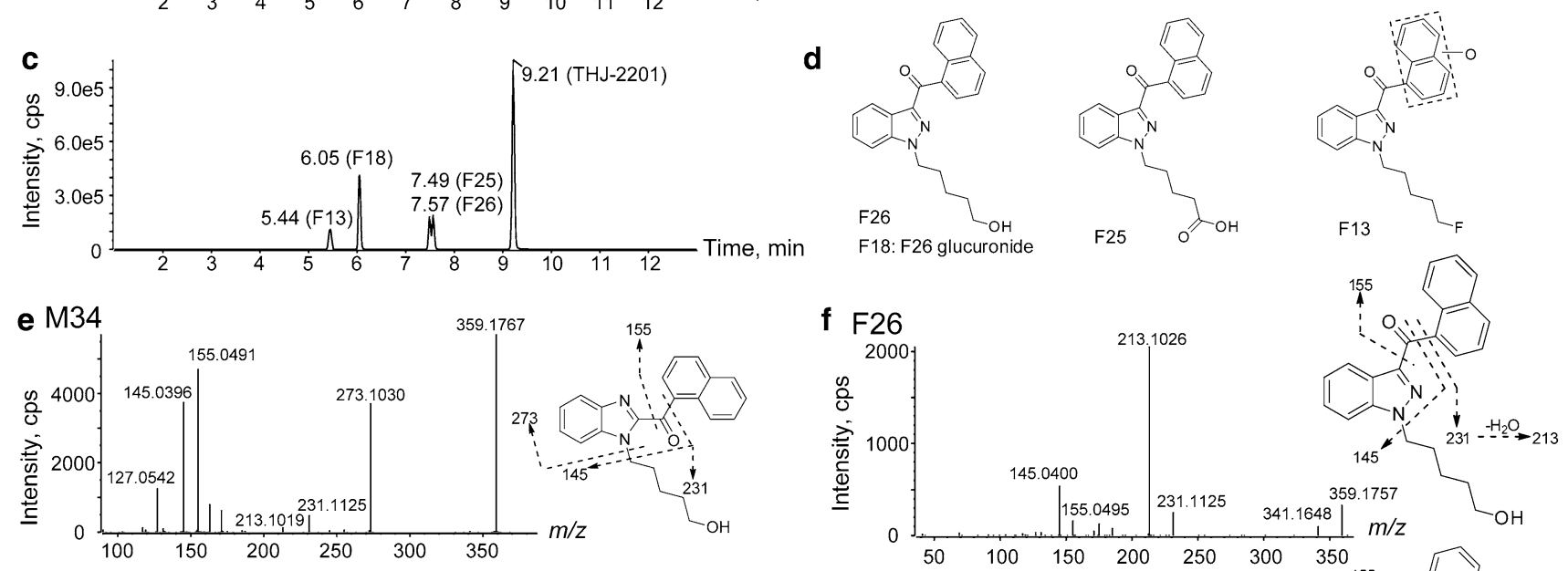

g M33

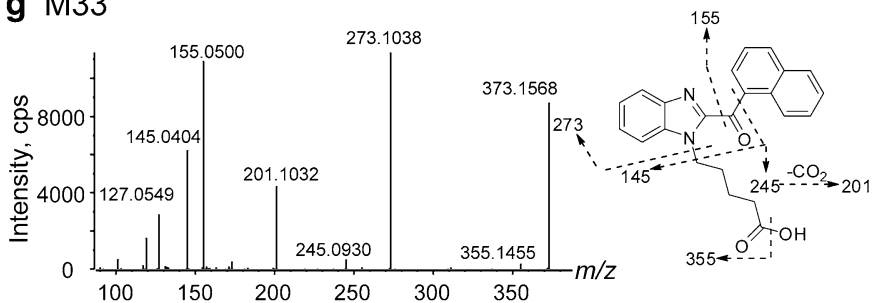

\section{h F25}
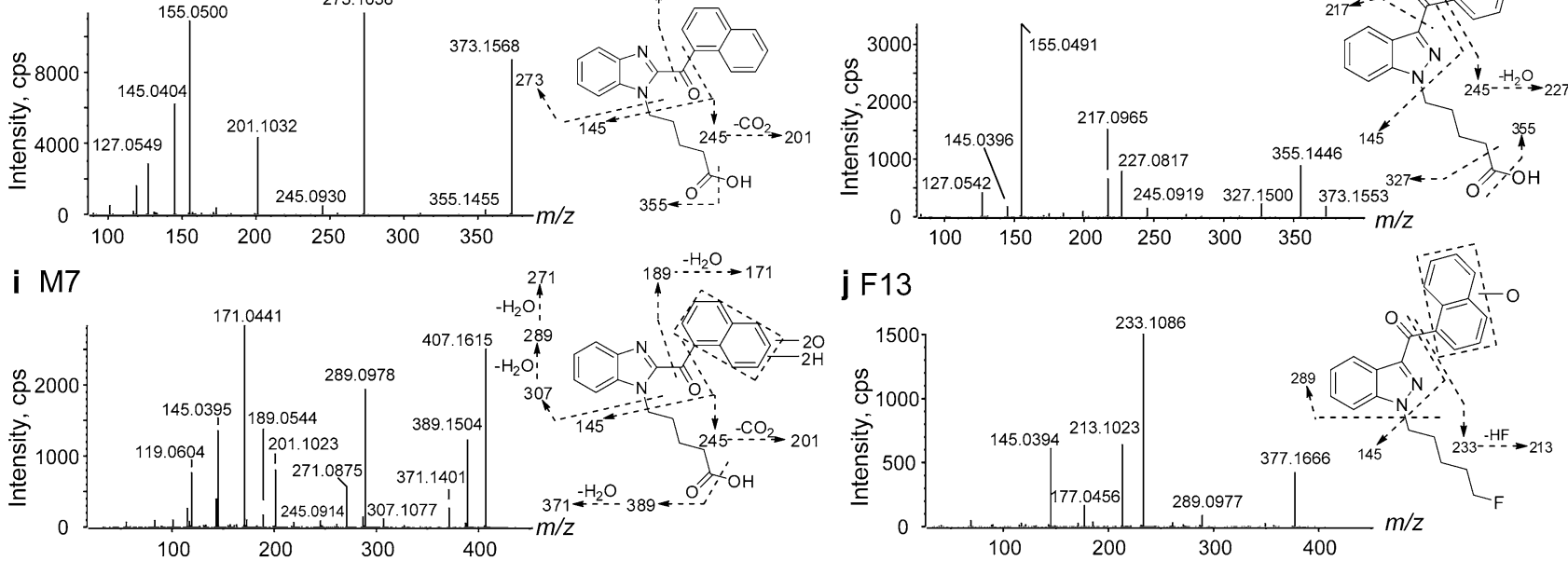

Fig. 6 Profiles of marker metabolites for FUBIMINA (a) and THJ-2201 (c); structures of three metabolite markers for FUBIMINA (b) and THJ2201 (d), and their product ion spectra and proposed fragmentation (e M34; f F26; g M33; h F25; i M7; j F13)

\section{Conclusions}

In summary, we characterized FUBIMINA metabolism in human hepatocytes with HR-MS. We recommend 5'-OHBIM-018 (M34), BIM-018 pentanoic acid (M33), and BIM-018 pentanoic acid dihydrodiol (M7) as the most promising urinary marker metabolites for documenting FUBIMINA intake. Their retention times and unique product ion spectra facilitate differentiation of FUBIMINA from THJ-2201 for clinical and forensic scientists. The data will enable linkage of potential adverse toxicological events to FUBIMINA or THJ-2201, in order that the public can be informed of their toxicity. These data also empower manufacturers to focus their synthesis efforts on optimal metabolites. We suggest workflow to differentiate isomeric SCs as follows: incubate SC isomers in human hepatocytes, analyze incubation samples by HPLC-HR-MS, characterize unique major metabolites' retention times and fragmentation profile, and confirm with case urine samples. Our strategies are applicable for distinguishing other SC isomers with different legal status. 
Acknowledgments This research is supported by the Intramural Research Program of the National Institute on Drug Abuse, National Institutes of Health. FUBIMINA was generously donated by the Drug Enforcement Administration, Department of Justice, USA.

\section{Compliance with ethical standards}

Conflict of interest The authors declare that they have no conflict of interest.

Ethical approval This article does not contain any studies with human participants or animals performed by any of the authors.

\section{References}

1. Castaneto MS, Gorelick DA, Desrosiers NA, Hartman RL, Pirard S, Huestis MA (2014) Synthetic cannabinoids: epidemiology, pharmacodynamics, and clinical implications. Drug Alcohol Depend 144:12-41

2. Patton AL, Seely KA, Chimalakonda KC, Tran JP, Trass M, Miranda A, Fantegrossi WE, Kennedy PD, Dobrowolski P, Radominska-Pandya A, McCain KR, James LP, Endres GW, Moran JH (2013) Targeted metabolomic approach for assessing human synthetic cannabinoid exposure and pharmacology. Anal Chem 85:9390-9399

3. Wiley JL, Marusich JA, Lefever TW, Antonazzo KR, Wallgren MT, Cortes RA, Patel PR, Grabenauer M, Moore KN, Thomas BF (2015) AB-CHMINACA, AB-PINACA, and FUBIMINA: affinity and potency of novel synthetic cannabinoids in producing $\Delta^{9}$-tetrahydrocannabinol-like effects in mice. J Pharmacol Exp Ther 354:328-339

4. Tait RJ, Caldicott D, Mountain D, Hill SL, Lenton S (2016) A systematic review of adverse events arising from the use of synthetic cannabinoids and their associated treatment. Clin Toxicol 54:1-13

5. Wilkinson SM, Banister SD, Kassiou M (2015) Bioisosteric fluorine in the clandestine design of synthetic cannabinoids. Aust $\mathbf{J}$ Chem 68:4-8

6. Hermanns-Clausen M, Kneisel S, Szabo B, Auwärter V (2013) Acute toxicity due to the confirmed consumption of synthetic cannabinoids: clinical and laboratory findings. Addiction 108:534-544

7. Seely KA, Lapoint J, Moran JH, Fattore L (2012) Spice drugs are more than harmless herbal blends: a review of the pharmacology and toxicology of synthetic cannabinoids. Prog Neuropsychopharmacol Biol Psychiat 39:234-243

8. Shanks KG, Winston D, Heidingsfelder J, Behonick G (2015) Case reports of synthetic cannabinoid XLR-11 associated fatalities. Forensic Sci Int 252:e6-e9

9. Tse R, Kodur S, Squires B, Collins N (2014) Sudden cardiac death complicating acute myocardial infarction following synthetic cannabinoid use. Intern Med J 44:934-936

10. Uchiyama N, Shimokawa Y, Kikura-Hanajiri R, Demizu Y, Goda Y, Hakamatsuka T (2015) A synthetic cannabinoid FDU-NNEI, two $2 \mathrm{H}$-indazole isomers of synthetic cannabinoids AB-CHMINACA and NNEI indazole analog (MN-18), a phenethylamine derivative $\mathrm{N}$-OH-EDMA, and a cathinone derivative dimethoxya-PHP, newly identified in illegal products. Forensic Toxicol 33:244-259

11. Uchiyama N, Shimokawa Y, Matsuda S, Kawamura M, KikuraHanajiri R, Goda Y (2014) Two new synthetic cannabinoids, AM2201 benzimidazole analog (FUBIMINA) and (4-methylpiperazin1-yl)(1-pentyl-1 $H$-indol-3-yl)methanone (MEPIRAPIM), and three phenethylamine derivatives, 25H-NBOMe 3,4,5-trimethoxybenzyl analog, 25B-NBOMe, and 2C-N-NBOMe, identified in illegal products. Forensic Toxicol 32:105-115

12. DeRuiter J, Smith FT, Abdel-Hay K, Clark CR (2014) Analytical differentiation of 1-alkyl-3-acylindoles and 1-acyl-3-alkylindoles: isomeric synthetic cannabinoids. Anal Chem 86:3801-3808

13. Scheidweiler KB, Jarvis MJ, Huestis MA (2015) Nontargeted SWATH acquisition for identifying 47 synthetic cannabinoid metabolites in human urine by liquid chromatography-high-resolution tandem mass spectrometry. Anal Bioanal Chem 407:883-897

14. Diao X, Scheidweiler KB, Wohlfarth A, Pang S, Kronstrand R, Huestis MA (2016) In vitro and in vivo human metabolism of synthetic cannabinoids FDU-PB-22 and FUB-PB-22. AAPS J 18:455-464

15. Diao X, Wohlfarth A, Pang S, Scheidweiler KB, Huestis MA (2016) High-resolution mass spectrometry for characterizing the metabolism of synthetic cannabinoid THJ-018 and its 5-fluoro analog THJ-2201 after incubation in human hepatocytes. Clin Chem 62:157-169

16. Wen B, Zhu M (2015) Applications of mass spectrometry in drug metabolism: 50 years of progress. Drug Metab Rev 47:71-87

17. Gao R, Li L, Xie C, Diao X, Zhong D, Chen X (2012) Metabolism and pharmacokinetics of morinidazole in humans: identification of diastereoisomeric morpholine $N^{+}$-glucuronides catalyzed by UDP glucuronosyltransferase 1A9. Drug Metab Dispos 40:556-567

18. Diao X, Deng P, Xie C, Li X, Zhong D, Zhang Y, Chen X (2013) Metabolism and pharmacokinetics of 3- $n$-butylphthalide (NBP) in humans: the role of cytochrome P450s and alcohol dehydrogenase in biotransformation. Drug Metab Dispos 41:430-444

19. Zhu M, Ma L, Zhang H, Humphreys WG (2007) Detection and structural characterization of glutathione-trapped reactive metabolites using liquid chromatography-high-resolution mass spectrometry and mass defect filtering. Anal Chem 79:8333-8341

20. Soars MG, McGinnity DF, Grime K, Riley RJ (2007) The pivotal role of hepatocytes in drug discovery. Chem Biol Interact 168:2-15

21. Baranczewski P, Stanczak A, Sundberg K, Svensson R, Wallin A, Jansson J, Garberg P, Postlind H (2006) Introduction to in vitro estimation of metabolic stability and drug interactions of new chemical entities in drug discovery and development. Pharmacol Rep 58:453-472

22. Diao X, Pang X, Xie C, Guo Z, Zhong D, Chen X (2014) Bioactivation of 3-n-butylphthalide via sulfation of its major metabolite 3-hydroxy-NBP: mediated mainly by sulfotransferase 1A1. Drug Metab Dispos 42:774-781

23. Wohlfarth A, Castaneto MS, Zhu M, Pang S, Scheidweiler KB, Kronstrand R, Huestis MA (2015) Pentylindole/pentylindazole synthetic cannabinoids and their 5-fluoro analogs produce different primary metabolites: metabolite profiling for AB-PINACA and 5F-AB-PINACA. AAPS J 17:660-677

24. Castaneto MS, Wohlfarth A, Pang S, Zhu M, Scheidweiler KB, Kronstrand R, Huestis MA (2015) Identification of AB-FUBINACA metabolites in human hepatocytes and urine using highresolution mass spectrometry. Forensic Toxicol 33:295-310

25. Ellefsen KN, Wohlfarth A, Swortwood MJ, Diao X, Concheiro M, Huestis MA (2016) 4-Methoxy- $\alpha$-PVP: in silico prediction, metabolic stability, and metabolite identification by human hepatocyte incubation and high-resolution mass spectrometry. Forensic Toxicol 34:61-75

26. Swortwood MJ, Carlier J, Ellefsen KN, Wohlfarth A, Diao X, Concheiro-Guisan M, Kronstrand R, Huestis MA (2016) In vitro, in vivo and in silico metabolic profiling of $\alpha$-pyrrolidinopentiothiophenone, a novel thiophene stimulant. Bioanalysis 8:65-82

27. McNaney CA, Drexler DM, Hnatyshyn SY, Zvyaga TA, Knipe JO, Belcastro JV, Sanders M (2008) An automated liquid 
chromatography-mass spectrometry process to determine metabolic stability half-life and intrinsic clearance of drug candidates by substrate depletion. Assay Drug Dev Technol 6:121-129

28. Diao XX, Zhong K, Li XL, Zhong DF, Chen XY (2015) Isomerselective distribution of 3-n-butylphthalide (NBP) hydroxylated metabolites, 3-hydroxy-NBP and 10-hydroxy-NBP, across the rat blood-brain barrier. Acta Pharmacol Sin 36:1520-1527

29. Obach RS (1999) Prediction of human clearance of twenty-nine drugs from hepatic microsomal intrinsic clearance data: an examination of in vitro half-life approach and nonspecific binding to microsomes. Drug Metab Dispos 27:1350-1359

30. Lave T, Dupin S, Schmitt C, Valles B, Ubeaud G, Chou RC, Jaeck D, Coassolo P (1997) The use of human hepatocytes to select compounds based on their expected hepatic extraction ratios in humans. Pharmaceut Res 14:152-155

31. Zhong S, Jones R, Lu W, Schadt S, Ottaviani G (2015) A new rapid in vitro assay for assessing reactivity of acyl glucuronides. Drug Metab Dispos 43:1711-1717

32. Uldam HK, Juhl M, Pedersen H, Dalgaard L (2011) Biosynthesis and identification of an $\mathrm{N}$-oxide/ $\mathrm{N}$-glucuronide metabolite and first synthesis of an N-O-glucuronide metabolite of Lu AA21004. Drug Metab Dispos 39:2264-2274

33. Ibrahim H, Couderc F, Perio P, Collin F, Nepveu F (2013) Behavior of $\mathrm{N}$-oxide derivatives in atmospheric pressure ionization mass spectrometry. Rapid Commun Mass Spectrom 27:621-628

34. Ismail IM, Dear GJ, Roberts AD, Plumb RS, Ayrtont J, Sweatman BC, Bowers GD (2002) $\mathrm{N}$-O glucuronidation: a major human metabolic pathway in the elimination of two novel anticonvulsant drug candidates. Xenobiotica 32:29-43

35. Freijo TD Jr, Harris SE, Kala SV (2014) A rapid quantitative method for the analysis of synthetic cannabinoids by liquid chromatography-tandem mass spectrometry. J Anal Toxicol 38:466-478

36. Skopp G, Potsch L (2002) Stability of 11-nor- $\Delta^{9}$-carboxy-tetrahydrocannabinol glucuronide in plasma and urine assessed by liquid chromatography-tandem mass spectrometry. Clin Chem 48:301-306

37. Scheidweiler KB, Schwope DM, Karschner EL, Desrosiers NA, Gorelick DA, Huestis MA (2013) In vitro stability of free and glucuronidated cannabinoids in blood and plasma following controlled smoked cannabis. Clin Chem 59:1108-1117

38. Li XD, Xia SQ, Lv Y, He P, Han J, Wu MC (2004) Conjugation metabolism of acetaminophen and bilirubin in extrahepatic tissues of rats. Life Sci 74:1307-1315

39. Gao C, Zhang H, Guo Z, You T, Chen X, Zhong D (2012) Mechanistic studies on the absorption and disposition of scutellarin in humans: selective OATP2B1-mediated hepatic uptake is a likely key determinant for its unique pharmacokinetic characteristics. Drug Metab Dispos 40:2009-2020

40. Xie C, Zhou J, Guo Z, Diao X, Gao Z, Zhong D, Jiang H, Zhang L, Chen X (2013) Metabolism and bioactivation of famitinib, a novel inhibitor of receptor tyrosine kinase, in cancer patients. Br J Pharmacol 168:1687-1706 